\title{
A plea for realistic assumptions in economic modelling
}

\author{
Leonardo IVAROLA
}

Received: 07/10/2017

Final version: $03 / 03 / 2018$

BIBLID 0495-4548(2018)33:3p.417-433

DOI: $10.1387 /$ theoria. 18133

ABSTRACT: The use of unrealistic assumptions in Economics is usually defended not only for pragmatic reasons, but also because of the intrinsic difficulties in determining the degree of realism of assumptions. Additionally, the criterion used for evaluating economic models is associated with their ability to provide accurate predictions. This mode of thought involves — at least implicitly - a commitment to the existence of unvarying invariant factors or regularities. Contrary to this, the present paper presents a critique to the use of invariant knowledge in economics. One reason for this analysis lies in the fact that economic phenomena are not compatible with the logic of invariance, but with the logic of "possibility trees" or "open-ended results". The other reason is that the use of invariant knowledge may entail both external validity problems and negative exposures to a "black swan". Alternatively, an approach where models are understood as possible scenarios is proposed. It is argued that the realism of (substantive) assumptions is crucial here, since it helps to ascertain the degree of resemblance between the different models and the target system.

Keywords: Milton Friedman, economic models, invariance, assumptions, prediction.

RESUMEN: En economía el uso de supuestos irrealistas se defiende generalmente no sólo por razones pragmáticas, sino también por las dificultades intrínsecas para determinar el grado de realismo de los supuestos. Asimismo, el criterio utilizado para evaluar los modelos económicos está asociado con su capacidad para proporcionar predicciones precisas. Este modo de pensamiento implica, al menos implícitamente, un compromiso con la existencia de regularidades invariantes. Al contrario de esto, el presente artículo presenta una crítica al uso del conocimiento invariante en economía. Una razón para este análisis reside en el hecho de que los fenómenos económicos no son compatibles con la lógica de la invarianza, sino con la lógica de los "árboles de posibilidades" o "resultados de final abiertos". La otra razón es que el uso del conocimiento invariante puede implicar tanto problemas de validez externa como exposiciones negativas a un "cisne negro". Alternativamente, se propone un enfoque donde los modelos se entiendan como escenarios posibles. Se argumenta que el realismo de los supuestos (sustantivos) es crucial aquí, ya que ayuda a determinar el grado de semejanza entre los diferentes modelos y el sistema objetivo.

Palabras clave: Milton Friedman, modelos económicos, invarianza, supuestos, predicción.

\section{Introduction}

The debate about the realism of assumptions in economic models reached one of its highest points with Milton Friedman's essay "The methodology of positive economics", where he examined the nature of economic models and their usefulness for explanatory and predictive purposes. Against the "naive" realistic approaches which defended the idea of evaluating eco- 
nomic models through the realism of their assumptions, Friedman sought to specify what kind of statements assumptions are, what their role is in the structure of a model, how both model and assumptions are evaluated, etcetera. In his analysis, Friedman ends up advocating-as most authors consider-an "instrumentalist" conception of economic science, since its task is to provide "a system of generalizations that can be used to make correct predictions about the consequences of any change in circumstances" (Friedman 1953, 4).

Friedman's essay has produced an enormous impact on economic methodology, and his defense of the unrealism of assumptions indicates the existence of important philosophical problems. More precisely, Friedman argues that in a strict sense all assumptions are unrealistic, since they involve idealizations and abstractions. Therefore, asking the right question is not about the realism of assumptions (for they never are), but about their approximation to reality. To such controversy, Friedman responds that the only way to know whether approximations are good or not is by seeing the predictive accuracy of the model implications. This means that the test of the model assumptions and the test of the model implications cannot be separated. There is only one test: the empirical test of the model implications.

This paper undertakes a critique to Friedman's approach. On the one hand, it is asserted that Friedman's defense of the unrealism of assumptions only makes sense as long as economic models account for invariant regularities. However, this is not the case. In economic phenomena the causal link between variables are people's actions. Because such activities depend on how people interpret signals from the world, how they form their expectations, how their decisions are influenced by cultural and institutional factors, etcetera, economic processes may fluctuate in unexpected ways. For this reason, it is argued that economic phenomena do not respond to a logic of stable factors or invariant regularities, but to a logic of possibility trees or open-ended results.

In addition to this, there is an epistemological problem that arises from the use of invariant regularities: even when they are identified in the economic realm, there is no guarantee that such stability will prevail in the future. It is shown that predictions based on knowledge from the past involve not only external validity problems but also negative exposures to "black swans". An alternative approach is proposed here where models are evaluated, not by their degree of invariance or the accuracy of their (past) predictions, but by their resemblance to a selected part of the world (or target system) about which predictions are made. Since the core structure of models depends on their substantive assumptions and since what is intended in this approach is to find a structural resemblance between a model and a target system, it is argued that the realism of substantive assumptions is crucial for the assessment and subsequent choice of economic models.

However, this approach is inconsistent with Friedman's idea of testing assumptions according to a model's implications. In this juncture, the first critique to Friedman's approach consists of showing that, as long as other alternative assumptions are taken into account, model assumptions can be tested independently of the model's implications. In this regard, it is argued that there are at least three kinds of questions one may ask in relation to the realism of assumptions. The first is to ask whether or not an assumption $S$ is realistic. The answer is always negative: all assumptions are unrealistic, since they are all simplifications and/or abstractions of reality. The second is to ask whether or not $S$ is a good approximation to reality, which—as Friedman said—can only be answered through the accuracy of implications. The third question — not taken into account in Friedman's analysis-is to 
ask whether an assumption $S$ is more or less realistic (or more or less approximate to reality) than another assumption $S^{*}$. It is argued that the answer to such questions does not depend on the test of the model's implications.

\section{Unrealistic assumptions in economic models: Friedman's approach}

As a positive science, Friedman advocates a limited notion of the goals of economics. According to the author, what is really interesting of a model or hypothesis is its ability to provide "a system of generalizations that can be used to make correct predictions about the consequences of any change in circumstances" (Friedman 1953, 4). In this regard, Friedman asserts that evaluating economic models by testing their assumptions is a methodological fallacy. In economics there is an important debate about the realism of assumptions. Heterodox economists consider that economic models should be analyzed and criticized not for their predictions but for their assumptions. This is exactly what Friedman questions. In the strict sense of the word, all assumptions are unrealistic: they are all simplifications and/or abstractions taken from reality. It is not possible to give an exhaustive description of the real world; nor is that necessary. On the contrary, it is worthwhile saying "a lot" with "little". The point is that if we want to achieve this goal, theories must be unrealistic. Thus, it does not seem to be adequate to ask about the realism of assumptions, because they never are realistic. What is important is that assumptions should be good approximations to reality, and the only way to know that is by examining a model' implications:

[...] the relevant question to ask about the "assumptions" of a theory is not whether they are descriptively "realistic," for they never are, but whether they are sufficiently good approximations for the purpose in hand. And this question can be answered only by seeing whether the theory works, which means whether it yields sufficiently accurate predictions. (Friedman 1953, 15)

Friedman uses the law of falling bodies as an example of his stance. That law claims that the acceleration of a body dropped in a vacuum is a constant $g$, and is independent of the shape of the body, the manner of dropping it, etcetera. The distance traveled by a falling body is given by the formula $s=1 / 2 \mathbf{g t}^{2}$, where $s$ is the distance and $t$ is the time. At sea level the air pressure is about 15 pounds per square inch. It is obvious that the assumption of a "vacuum" is not realistic, since 15 is different from zero. However, it is not relevant to demonstrate that there exists a difference between the assumed value and the real value, but rather to know if such a difference is significant or not, that is, if the assumption of vacuum is (or is not) a good approximation to reality. In this sense, Friedman argues that the only way to know this is through the accuracy of the predictions of the law. Let's suppose then that a heavy body (for example, a solid steel ball) is dropped from the roof of a building. How do we know if the assumption of vacuum is (or is not) a good approximation to reality? Through the comparison between the predicted value and the real value. In this case, the falling time of the ball is very close to the time predicted by the equation. Therefore, the assumption of vacuum will be a good approximation to reality. On the other hand, if a feather is dropped from the roof instead of the solid ball, then the predicted value will be quite different from the real one, and so the assumption of vacuum will not be a good approximation to reality. 
If the above argument is true, then the testing of assumptions and the testing of predictions cannot be separated. There is only one test: the empirical test of the model's implications. In other words, it is not possible to directly estimate the discrepancy between what is asserted in the assumptions of a model and what is observed in the real world. Such a discrepancy is estimated indirectly through the model's implications. ${ }^{1}$ This is something that must not be overlooked. If the two tests are reduced to one test only, then defending the realism of assumptions is unsustainable, as there is no empirical way to know if an assumption is realistic or unrealistic.

Prediction, then, is all that matters. We should not make a literal reading of theories and models, but understand them from an "instrumentalist" point of view: it is not claimed that vacuum truly exists, but that some objects like a steel ball fall as if they were falling in a vacuum. Friedman suggests that economic hypothesis should be evaluated in a similar manner. According to him, critics of the maximization-of-returns hypothesis are ill-founded, because they assess the realism of the assumptions involved, when in fact they should assess whether there are significant discrepancies between the predicted results and the phenomena observed. It is not that firms produce at the point where marginal revenue equals marginal cost, but they behave as if they did.

Critiques of Friedman's approach soon appeared, ${ }^{2}$ and they focused principally on the kind of assumptions used in building an economic model. One of these was postulated by Nagel (1963), who distinguished among three types of assumptions: as non-exhaustive descriptions, as abstractions and as falsehoods. Nagel considers that the first two kinds are not problematic; yet this is not true of the third kind. Similarly, Musgrave (1981) criticizes Friedman's approach for not being able to distinguish between three different types of assumptions: negligibility, heuristic and domain assumptions. Each of these makes a different type of assertion and therefore plays a different role in economic models. More recently, Rodrik (2015) argues that the realism of a particular sort of assumptions is relevant for economic analysis. Specifically, he says that we always need to apply a realism filter to "critical assumptions" before a model can be considered useful. An assumption is critical "if its modification in an arguably more realistic direction would produce a substantive difference in the conclusion produced by the model" (Rodrik 2015, 27). Many assumptions are not critical in this sense. As a consequence, we should not pretend that a model is completely realistic, but the realism should be focused on those assumptions that make a difference in a model's results. Let us take for instance the general equilibrium theory. Whether agents are fully rational, possess full information or have long term horizons is not of much interest. However, there are critical assumptions such as the shape of the demand curve, whether firms have market power or not, etc. These assumptions make a significant contribution to the results, in the sense that changes in them lead to changes in the model results. Because of that, they cannot be unrealistic. In a similar analysis, Kuorikoski and Lehtinen (2009) state that theoretical modeling usually involves two types of assumptions: "auxiliary" and "substantive”. Substantive assumptions concern aspects of the model's central causal mechanism

1 “[...] a theory cannot be tested by comparing its 'assumptions' directly with 'reality'. Indeed, there is no meaningful way in which this can be done.” (Friedman 1953, 41)

2 Given the extent of the critiques to Friedman's approach, only a small sub-group of these contributions will be mentioned in the present paper. For a more complete list, see Hands (2001). 
about which one makes important assertions. On the other hand, auxiliary assumptions are required for making inferences from substantive assumptions to conclusions feasible. In other words, auxiliary assumptions play the heuristic role of making the model more tractable. Kuorikoski and Lehtinen (2009) believe that while it is true that auxiliary assumptions create different kinds of distortions and biases in model inferences, such distortions may be "removed" by using derivational robustness analysis. Therefore, the unrealism of auxiliary assumptions is not put into question. Nevertheless, it is necessary that substantive assumptions be realistic, so that they may carry their epistemic weight into the results. Otherwise, robustness analysis would be useless.

\section{The test of assumptions versus the test of predictions}

Critiques to Friedman's approach take for granted the feasibility of testing the model assumptions. However, this is precisely what Friedman put into question. How do we know if a substantive assumption is credible? How do we know if a domain assumption is false? The answer is: Through the accuracy of a model'spredictions. This is the only way to know if an assumption is a good approximation to reality. For Friedman, if a model provides good predictions-or "significant" ones, in Musgrave's terms-then we can say that its assumptions are good approximations to reality. It is not that some models are not significant because their assumptions are bad approximations, but that assumptions are bad approximations precisely because models are not significant (that is, they do not provide accurate predictions). Thus, from an instrumentalist point of view, it would not only be unimportant to incorporate assumptions that are congruent with real phenomena, but in practice, as there is no independent way for determining such congruency, it is not possible either to estimate their relevance until the model's predictions have been previously examined.

In economics, discussions associated with the realism of assumptions have provoked a divide between scholars who advocate testing models through the accuracy of their assumptions and those who advocate testing models through the accuracy of their predictions. Mäki (2009b) has called the former assumptionists and the latter predictivists. Assumptionists presuppose the feasibility of testing assumptions "directly", that is, without paying attention to the model's predictions. In contrast, predictivists assert that it is not possible to test an assumption directly, but only indirectly through its predictive implications: "when one seeks to test an assumption, one has to construe an argument in which that assumption serves as one of the major premises and which entails a predictive implication that one then compares with evidence" (Mäki 2009b, 96).

However, the distinction Mäki makes between these two groups is not necessarily associated with an ideological difference, but rather with a "technological" difference. According to Hempel (1966), there are some occasions in which the testing procedure is quite direct (for example, statements such as "it's raining outside" or "the cat is under the table"). However, many scientific hypotheses cannot be tested directly, so that indirect methods of testing must be carried out. In cases like these, the test is based on an argument to the effect that if the statement were true, then certain observable events should occur under specified circumstances. What is tested is not what the statement says but what it implies. An example of this is the hypothesis that the Earth is round. Aristotle used lunar eclipses to test that the Earth was not flat but spherical. In this case the test is clearly indirect. Aristotle had 
to create an argument by which, if the hypothesis were true, certain results should be observed. Nevertheless, this does not necessarily imply that testing is always indirect. In outer space an astronaut does not need to create an argument for testing the hypothesis; he may simply observe the spherical shape of the earth through the bull's eye. Indirect testing is much more common than direct testing, yet it does not follow from this that it is not possible to test a hypothesis directly. ${ }^{3}$

However, Friedman — one of the main "predictivists" — seems to go beyond the mere discussion about testing assumptions directly or indirectly. He claims that, in fact, the test of assumptions cannot be carried out without testing a model's implications; in other words, the test of the model's assumptions is not independent from the test of the model's implications. In order to explain the difference between what is indirect and what is independent, let us suppose a model $\mathrm{M}$ from which a result $\mathrm{R}$ is inferred. Let us also consider a set of assumptions S1, S2 and S3 that belong to M. We may consider M the "argument" for testing S1, S2 and S3. According to Friedman, the only way to know if that set of assumptions is a good approximation to reality is by testing the accuracy of R. Let us now suppose that we only want to test assumption S1. Friedman will say that the only way to do that is to test the accuracy of R. Predictivists, on the other hand, will assert that we must necessarily create an argument for testing S1. M may be a possible argument, yet it is not the only one. We might create a new argument A for testing S1. Let $\mathrm{R}^{*}$ be the result inferred from A. What is important to predictivists is the accuracy of $\mathrm{R}^{*}$. Let's take for example Galileo's law and its assumption of vacuum. One way to evaluate the realism of this assumption is by testing Galileo's law itself. But that is not the only way; we could also design a Torricelli experiment to measure atmospheric pressure.

The latter example allows us to show that the test of S1 can be independent from the test of M. In general, one thing is to say that what is tested are the implications of a statement (indirect test), and quite another thing is to say that the test of the model's assumptions is not independent of the test of the model's predictions. In this juncture, a deeper analysis allows us to show that this problem depends on the type of question we ask in relation to the realism of the assumptions. Let $\mathrm{X}$ and $\mathrm{Y}$ be different assumptions. Three types of questions can be asked:

- Is $\mathrm{X}$ a realistic assumption?

- Is $\mathrm{X}$ a good approximation to reality?

- Is X more realistic than Y?

Friedman analyzes the first two types of questions, but he leaves out the third possibility. For the sake of clarification, let us return to the example of the law of falling bodies. Such a law is valid for bodies falling in a vacuum, so in order to test the law we might previously measure air pressure. At sea level the air pressure is approximately 15 pounds per square inch (psi). Then the first type of question stated above may be asked: Is the assumption of vacuum unrealistic? Clearly it is, since 15 is different from zero. However, Friedman asseverates that this question has no meaning because, in a strict sense, all assumptions are unre-

3 In no way does it mean that the difference between the two tests is something that can be solved with more technology. Technology can help in some cases, but not always. Regardless, what is important to note, contrary to what Mäki asseverates, is that empirical tests are not always indirect. 
alistic. According to Friedman, what is right is asking whether the 15 psi is close enough to zero for the difference to be judged insignificant. The only way to know that is by testing the accuracy of the predictions of Galileo's law.

As long as the second type of question is formulated, there will be no independence between the test of the model's assumptions and the test of the model's predictions. However, this is not true when the third type of question is asked: “is X more realistic than Y?". If we wonder whether the assumption of vacuum in Galileo's law is a good approximation to reality, we must necessarily test the implications of this law. However, we may go one step further and ask whether the assumption of vacuum is more or less realistic than any other assumption, say, one that posits an atmospheric pressure of 15 psi. Here, Galileo's lawand its respective implications-loses relevance. What is being asked now is: "which of the two statements comes closer to reality: 0 psi or 15 psi?”, so that a new argument for testing both statements must be constructed. This means that the experiment conducted for measuring atmospheric pressure (also known as "Torricelli's experiment") is independent from the experiment conducted for testing Galileo's law.

Thus, not only is it possible to test the assumption of vacuum without testing the implications of Galileo's law, but it is also empirically possible to prove that the assumption of vacuum is less "realistic" —or at least less approximative to reality-than the assumption of 15 psi. It is likely that due to changes in climatic conditions air pressure is not exactly 15 psi. However, what can be said with confidence is that, in the proximity of sea level, the assumption of 15 psi will be a lot closer to reality than the assumption of vacuum, and said result is reached without resorting to the implications of Galileo's law. In a more general level, we may say that model assumptions (or at least most of them) are independently testable from the testing of the model's implications. This is achieved when we change the question "Is X a good approximation to reality?" for the question "Is X more realistic than Y?".

The question whether real entrepreneur decision-making is better explained by psychological heuristics or by microeconomic theorems is therefore not necessarily something that has to be decided on the basis of the predictive accuracy of models. The same applies to many other assumptions in economics. Even if the testing of any statement is almost always indirect, it is not correct to assert that the testing of model assumptions is not independent from the testing of model predictions. It is likely that some economists advocate for the unrealism of assumptions. However, this attitude would only be justified by pragmatic preferences and not because the testing of the model's assumptions is impossible to perform.

\section{The need for invariant regularities in Friedman's approach}

Scientists use data to develop theories and models. In some occasions such data is used as a background to formulate models that are expected to provide accurate and novel predictions. However, in other situations data is used exclusively to "accommodate" models to data. This juncture has led to the discussion "prediction versus accommodation". Extreme positions are usually criticized. For example, Hitchcock and Sober (2004) assert that excessive accommodation leads to the problem of "overfitting", which ends up undermining the very goal of predictive accuracy. On the other hand, focusing exclusively on novel predictions may cause that relevant information is not used in formulat- 
ing theories and models. Beyond this discussion, both approaches seem to share the idea that what really matters is that the association between independent and dependent variables-or between inputs and outputs-should be stable or invariant. If a theory does not predict accurately, the scientist may accommodate it so that the association between inputs and outputs is invariant. Analogously, a model that provides accurate predictions is similar to saying that the discrepancy between the predicted value and the real value is not significant, which is similar to saying that the relation between a model's inputs and outputs is stable or invariant.

In some way this principle can also be found in Friedman's approach. According to Friedman, the task of positive economics is to provide a system of generalizations that can be used for making accurate predictions. This accuracy is the basis for accepting or rejecting models, in the sense that the models that provide more accurate predictions will be preferred over others. On the other hand, predictive accuracy depends on the success of a model in previous circumstances. Thus, Friedman seems to be advocating an approach in which scientific progress goes hand in hand with the discovery of stable or "invariant" regularities. ${ }^{4}$ Such an investigative practice can be illustrated as follows: Let us suppose three models M1, M2 and M3. Each model starts from the same initial situation X. However, they appeal to different mechanisms and background conditions, so different results $R$ are inferred: R1 for M1, R2 for M2 and R3 for M3. We then have three generalizations G1, G2 and G3 connecting the same initial situation X with R1, R2 and R3 respectively. Suppose that the relationship that has historically prevailed the most is between X and R1. G1 will be then the most invariant generalization. Also, since M1 is the model that has provided the most accurate predictions, Friedman would say that we should use said model for future predictions.

Thus, the criterion Friedman uses for choosing among different economic models seems to be the level of invariance of regularities. And the more invariant the regularity, the more "fruitful" the model. It does not matter if the assumptions of the model are realistic or unrealistic. What is important is that the relation between $X$ and $R$ is stable or invariant. It would also be plausible to say that the target system behaves as if what is stated in the model were true. Let's take Friedman's example of the hypothesis that plants maximize their reception of sunlight. As long as an invariant regularity between the growth of plants and the sunlight they receive is discovered, we could say that plants behave as if each leaf deliberately sought to maximize the amount of sunlight it receives. Based on this knowledge we will be able to predict - with a high degree of confidencewhat would happen under different circumstances. For instance, we may use this hypothesis for inferring that in the Northern hemisphere foliage is generally denser on the south side of trees, whereas in the Southern hemisphere foliage is generally denser on the north side of trees.

The requirement of invariant knowledge for predictive purposes may involve two types of assumptions: an ontological assumption which assumes the existence of stable factors, and an epistemological assumption in which it is assumed that invariant knowledge can be used in different situations. Some of these assumptions must be met so that the use of invariant knowledge is fruitful. On the one hand, we may assume an ontology of stable causal fac-

4 Other names for invariant regularities are "tendencies", “empirical generalizations", etc. 
tors in which, if those factors were properly isolated from perturbing factors, it would be possible to predict what will happen once the triggering factor is activated (see for example Cartwright 1999; Maki 1992). On the other hand, one could not stick to an ontological commitment, but rather to a weaker approach where the only requirement is observing invariant regularities at the level of events. Both assumptions are independent, in the sense that any of them may occur without the other. There is a chance that a stable causal factor be found in isolation, but at the level of events other factors end up disturbing such stability. Similarly, it is possible to find regularities which are not the manifest results of a stable causal factor (for example, those regularities connecting variables that come from a common cause). What really matters is that at least one of these assumptions be met so that an invariant-based approach of prediction makes sense.

Nevertheless, what follows shows that these assumptions are problematic to tackle the issue of prediction in economics. This section is divided into two parts: the first concerns the ontological assumption of stable causal factors, and the second refers to the epistemological assumption.

\subsection{Ontological ASSumption: THE EXistence of Stable CAUSAl FACTORS}

Regarding the ontological assumption, it is important to analyze the way in which economic variables are connected. Following the dualistic ontology proposed in Machamer, Darden and Craver (2000) and in Machamer (2004), these relationships are mediated by activities. More precisely, the authors distinguish between "entities" and "activities" in a mechanism. Activities are "the producers of change", while entities are "the things that engage in activities" (Machamer, Darden and Craver 2000, 3). For Machamer et al., activity is a "singular" notion: whenever a factor $F$ is triggered, an activity $A$ is involved in the production of a result R. Singular means that there are no other activities implicated between $F$ and $\mathrm{R}$. In some occasions, $\mathrm{R}$ may not follow from $\mathrm{F}$, yet this does not depend on the activity A but on perturbing factors. For instance, if we have a headache and the aspirin we take does not produce relief, it does not depend on the very activity the aspirin produces in our body. Acetylsalicylic acid inhibits the production of prostaglandins. This is the activity of aspirin. Every time we take an aspirin, the activity of inhibition starts to function. There are no other activities that replace it. That the aspirin failed to relieve the headache, does not mean that the activity of inhibition did not take place. In a general tendency, it did, but it was countered by perturbing factors.

Despite the fact that Machamer et al.'s approach is best suited for biology than for economics, it is useful to help us understand the workings of economic phenomena, because it highlights the notion of activity and its relationship with entities and/or variables. In this regard, economic activities are plainly the decisions of individuals. These activities are influenced by several factors such as those related to the socio-cultural sphere, the information agents receive from the world, the expectations they form about the evolution of certain variables, etcetera. Depending on what types of activities people carry out, different results will be observed in the economy. Therefore, contrary to Machamer et al.'s approach, economic activities are not singular but "plural": once a factor $F$ is triggered, a set of potential activities $\left(A_{1}, A_{2}, \ldots, A_{n}\right)$ may start to work. Each of these activities are involved in the production of a different result $\left(R_{1}, R_{2}, \ldots, R_{n}\right)$. The prevailing result will depend on how people form their expectations, how they interpret information from the economic and politi- 
cal world, the socio-cultural framework, the changes in the social and economic structures, etc. (Ivarola 2017).

Economic phenomena are then congruent with the logic of open-ended-results or possibility trees: given a certain event (inflation, increase in government expenditure, fall in real wages, etc.), there are several potential alternatives. Any of these, in principle, is plausible. The final result will depend on what activities people carry out. A good example of this is the "Keynes effect". It is a mechanism in which an increase in the real quantity of money leads to a decrease in the interest rate, stimulating investment and consequently the employment and the level of output. However, it is wrong to think that a positive change in the real quantity of money will invariably lead to higher levels of output. It is even implausible to think that changes in real quantity of money will invariably lead to a decrease in the interest rate. On the contrary, depending on the contextual framework and on people's expectations the course of the economy may be different. Keynes was explicit on this matter:

\footnotetext{
"[...] whilst an increase in the quantity of money may be expected, cet.par., to reduce the rate of interest, this will not happen if the liquidity-preferences of the public are increasing more than the quantity of money; and whilst a decline in the rate of interest may be expected, cet. par., to increase the volume of investment, this will not happen if the schedule of the marginal efficiency of capital is falling more rapidly than the rate of interest; and whilst an increase in the volume of investment may be expected, cet. par., to increase employment, this may not happen if the propensity to consume is falling off. Finally, if employment increases, prices will rise in a degree partly governed by the shapes of the physical supply functions, and partly by the liability of the wageunit to rise in terms of money. And when output has increased and prices have risen, the effect of this on liquidity-preference will be to increase the quantity of money necessary to maintain a given rate of interest." (Keynes 1936, 155)
}

The example clearly shows the lack of a "singular" invariant connection between economic variables. There is no causal force that induces people to demand more bonds whenever the quantity of money is increased. Depending on the context they may buy goods, hoard the extra money, etc. Activities must be understood in a potential sense, since any of them may take place. It is important to point out, however, that the concept of "causality" (in the sense of productive relationships between entities or variables) is not under debate. It is recognized that the real amount of money may bring about an increase in the GDP. Nevertheless, it may bring about other results (for example, inflation). The problem is not causality, but the invariance or stability of causal factors.

\subsection{EPistemological asSUmption: THE EXISTENCE OF INVARIANT KNOWLEDGE AND ITS USE FOR DIFFERENT PURPOSES}

Although economic phenomena may be understood through the logic of open-ended results, it does not follow from this that in some occasions economic tendencies may be observed at the level of events. If the price of beef rises $500 \%$, people have the option of consuming it or substituting for pork or poultry; yet, it is reasonable to expect an important decline in the consumption of beef. Similarly, when one sees a dog chasing pigeons in the park, there is the chance that they will stay put, escape or attack. But pigeons (almost) always end up escaping. This means that in many situations, even if 
there are many alternatives or courses of action available, there is a "dominant" course that prevails. The reasons can be attributed to several factors, for example, stability in the expectations formation process, institutional factors that do not change over time, etcetera. When this happens, it is likely to observe a tendency or regular behavior of some economic variable.

This latter case leads to the problem of an epistemological assumption, which is not associated with finding invariant knowledge but with what we do with it. In this juncture, Cartwright (2007) and Cartwright and Efstathiou (2011) have asserted that there are no troubles in "hunting" stable causal factors; the real problems arise when using such causes for different purposes (predictive, explanatory, etc.). In other words, there lacks a bridge to connect the discovery of causal factors with their respective use. Without this bridge, there is no warranty that a causal factor will work in different circumstances or scenarios, regardless of its level of invariance.

Broadly speaking, Cartwright and Efstathiou (2011) consider that the conditions that several accounts need to secure causal knowledge are not sufficient to secure the inferences that would put them to use. These accounts face two problems: Unstable enablers and External validity. The former is related to changes in the enabling factors that support invariant regularities. The latter is associated with the problem of generalizing from a particular setting or population to the one of interest.

The problem with unstable enablers is the fact that the contribution of a causal factor may be perturbed due to changes in background conditions in which that factor operates. This means that any effect of a particular cause depends on a set of other causal factors operating simultaneously, factors that are rarely easy to identify. Besides this problem in the background arrangement of causes, there is a second kind of unstable enablers: changes in the underpinning structures that give rise to economic regularities. According to this notion, an invariant regularity takes place precisely because there is a robust structure that supports it. However, the presence of one or more perturbing factors may alter the structure, thereby cancelling the invariant relationship.

The second problem is associated with the external validity of invariant regularities. There is external validity when the result obtained in some target system holds outside of that target. However, even if we establish results very securely in a particular test, the methods used in such situations provide no basis for extending the results to a population or setting different from those in the test. Because of this, the knowledge of invariant regularities is very narrow in scope and thus very limited in their predictive power. This problem can be illustrated with the "Galilean experiments". The goal in a Galilean experiment consists of eliminating all confounding factors in order to get an invariant regularity between cause and effect. Although we can eliminate confounding factors by physically isolating an experimental system from background interference and/or by making various idealizing assumptions, this does not ensure that an invariant causal relation will persist once confounders are present (Cartwright and Efstathiou 2011).

Taleb (2007), on the other hand, considers that invariant knowledge (or, better put, knowledge derived from data) is not only of little help in understanding both the present and the future, but also such an inductivist conception can be the reason for the appearance of "black swans". From an epistemological point of view, a black swan is an outlier, an event that lies outside the realm of regular expectations. Since it is a highly improbable event, it is impossible to predict. Also, it carries an extreme impact to such a degree that it ends up 
changing the course of history. ${ }^{5}$ A case that perfectly represents the notion of the black swan is the so called "Russell's inductivist turkey". Let us consider a turkey that is fed every day. Each additional feeding will firm up the bird's belief that it is the general rule of life to be fed every day by a farmer. However, the day before Thanksgiving, something unexpected will happen to the turkey, something that cannot possibly be predicted on the basis of that general rule. What the turkey has learned about what is in store for it tomorrow from the events of yesterday may be important, although certainly a little less than it thinks, and it is just that "little less" what may make all the difference (Taleb 2007).

The "black swan" metaphor comes from the widely ancient belief that all swans are white. Before the discovery of Australia the empirical evidence was irrefutable, and each additional empirical data increased the degree of confirmation of that universal statement. However, such discovery brought about a totally unthinkable surprise: the existence of swans with black plumage. It illustrates a severe limitation to our learning from observation and the fragility of knowledge based on empirical confirmation: one single observation can invalidate a general statement derived from confirmatory sightings of thousands of white swans. According to Taleb, in many situations what we do not know may be more important than what we know. Therefore, the theory of the black swan is a critique to the use of induction as a way of learning in general, and to the criterion of evaluating models according to their predictive accuracy in particular. In these cases, extreme events are considered absolute outliers and therefore are dismissed as a possible alternative. There is a tendency to understand the world from what is already known, that is, to use induction. However, knowledge of the past is not problematic per se; the problem is what we do with that knowledge. And what we usually do is dismiss the unknown, that what could happen even if it has never happened yet (just as the turkey dismisses the chance that the farmer will cut its head off). This way of thinking exposes people to black swans, and its consequences are often not only negative but also irreversible.

Invariance cannot then be the criterion for choosing among different economic models. A model providing more accurate predictions than others only gives us information about what has happened in the past. However, we have no basis to expect that such stability will continue in the future. To begin with, economic ontology is not about stable causal factors but about open-ended results. Likewise, even if a tendency is "hunted" in some situation, nothing guarantees that such a tendency will continue to hold in another context. Finally, whether we only focalize on what we know - so that the unknown is dismissed or considered a rarity - we are exposing ourselves to black swans. Therefore, and contrary to Friedman's approach (and of some "predictivists"), economic models should not be evaluated (at least only) by their predictive accuracy. Because of this, perhaps the discussion about the realism of assumptions is not as irrelevant as Friedman believed.

\section{Possible scenarios and the importance of using realistic assumptions}

A clear problem of appealing to invariance knowledge is becoming a Russell's inductivist turkey. Being a Russell's turkey means using past information for making forecasts. How-

5 Not all outliers are a black swan. In order to be one, the impact must be considerable. 
ever, without this information we are not able to make predictions (or at least this is what Friedman and his followers claim). This is partly true and partly false. It is true that we lose the ability to predict what economic events are precisely going to occur (for example, the turkey could not infer that the farmer will come to feed it tomorrow if he does not take into account the information of past feedings). However, it is false in the sense of losing the ability to make any kind of predictions. Despite the fact that many times it is difficult to compute probabilities to future events, it is not so complicated if we get a general idea about the possibility of their occurrence (as well as the turkey can get a general idea about the possibility that the farmer will eventually kill him).

To begin with, predictions based on the occurrence of possible events involve making use of more than one model. In each of these models a different set of substantive assumptions is specified. According to Kuorikoski and Lehtinen (2009), such kind of assumptions provide information about the core structure that leads to a result. Because in each model the set of substantive assumptions is different, it may be said that each of the models involved characterize a different scenario. The next step consists of finding a model whose structure resembles in some respects the structure of a selected part of the world (the "target system"). ${ }^{6}$ If that model exists, then it is feasible to think that the results of such a model will also occur in the target system. Therefore, the last step consists of choosing that model whose structure resembles more the structure of the target system. This means that examining the substantive assumptions of models is crucial in this procedure; otherwise, there would be no way to establish a choice criterion among different models.

In order to clarify this point, let us consider once again the Keynes effect example. Once the increase in the quantity of money takes place, Keynes proposes different possible scenarios. The most important scenario is the so called "Keynes effect": when the money supply is increased, a decrease in the interest rate will take place. This change will stimulate investment and consequently employment and production. For this to happen, certain conditions have to be met, namely, that there are constant returns to scale (so that prices do not rise or fall as output increases), the liquidity preference is not increasing (or at least such increase is lower than the increase in the quantity of money), the marginal efficiency of capital is not falling more rapidly than the interest rate, the marginal propensity to consume is not decreasing, etcetera.

However, Keynes is aware that other possible scenarios may happen. For instance, we may assume a second scenario where the liquidity preference grows more than the quantity of money. If so, then the monetary policy will have no impact on the interest rate. An interesting example of this is the "liquidity trap": if the interest rate is quite low, agents will be waiting for an increase in the interest rate. Therefore, they do not end up buying bonds, but prefer to hoard the surplus of money. Likewise, there is a third scenario where, although the monetary policy has successfully reduced the interest rate, entrepreneurs do not have good expectations about future sells. If so, then it is likely that the marginal efficiency of capital ends up falling more rapidly than the interest rate. In other words, firms will be

6 It does not mean that all economic models must be understood in this particular representational way. For instance, there are models whose main structure consists of a set of mathematical theorems without any connection with the real world. Because of this, this paper does not provide a general view of economic models, but only takes into account those models whose structure tells us something about the real world. 
reluctant to invest, even if the credits are cheaper. Keynes also considers a fourth scenario in which the rise of investment brings about an increase in primary employment in the investment industries (N2). Through Kahn's multiplier, the raise in N2 will produce a higher increase in total employment $(\mathrm{N})$. However, total employment will increase as long as firms producing consumer goods do not expect a drop in the marginal propensity to consume. If this happens (for instance, as a result of propaganda in time of war in favour of restricting individual consumption), then such firms would find no incentive to hire additional workers. Finally, as long as other economic theories are taken into account, new scenarios may be added to the analysis. For example, monetarists argue that if the quantity of money rises faster than the rate of growth of national income then there will be inflation. Keynesians also see a possible inflationary consequence in this monetary policy: if the increase in aggregate demand (caused by an increase in the quantity of money) ends up exceeding the level of full employment, then an increase in prices will take place.

Once the policy maker has these possible scenarios on hand, he must take a decision about whether or not it is adequate to implement a particular policy. He may appeal to invariant knowledge using the model which has provided the best results until now. However, we know that by doing this he would be acting like a typical Russell's turkey. The other criterion the policy maker may use is the approach defended in this paper: choosing the model whose structure is closer to the structure of the selected part of the world. Thus, if, for instance, the liquidity preference is high in the real world, it is likely that the increase in money supply will not be followed by a decrease in the interest rate. The model that better suits this situation will be the second scenario. On the other hand, even if there are no problems with liquidity preference, we can foresee that an increase in the quantity of money will have no results in national income as long as entrepreneurs do not have good expectations for future sales. Such a prediction is based on the information provided by the third scenario. Depending on the real world scenario, this monetary policy may not only fail to achieve its purpose (the increase in national income), but may also bring about undesirable effects. For instance, if the economy where the policy is implemented is close to full employment, then it is plausible that prices will indeed increase.

The logic of prediction proposed in this paper strongly differs from Friedman's approach in particular and from those approaches based on invariant knowledge in general. What is proposed here is not an approach based on the accuracy of models, but on the resemblance between the structure of the target system and the structure of the models considered. The accuracy of models is related to past information, so it may be of little help for future forecasts. On the contrary, what is important is to find a model whose structure resembles (as close as possible) the structure of the selected part of the world. Thus, whether or not the policy maker decides to increase the quantity of money, such a decision should not be based on past successes or failures of the policy, since the actual scenario may be substantially different from past scenarios. On the contrary, the decision should be based on the features of the target system.

Friedman relates the acceptance of a theory or model to its accuracy under a wide range of circumstances. Specifically, Friedman asserts that "under a wide range of circumstances, bodies that fall in the actual atmosphere behave as if they were falling in a vacuum" (1953, 18). Friedman also claims that "the formula is accepted because it works, not because we live in an approximate vacuum" $(1953,18)$. However, this is not true. The law does not work for a broad set of bodies thrown in free fall. There are many objects that when thrown 
in free fall will not behave according to Galileo's law. Unlike what Friedman states, the law itself refers to a physical principle that when any kind of resistance to free fall is eliminated it will be possible to observe its behavior in a pure state. Therefore, it does work in a vacuum. Some objects have a weight and a dimension such that in some way they crucially reduce air resistance. These objects, and only these ones behave as if they fell in a vacuum. Thus, if our purpose is to predict what will happen in some target system, we must know the background whereby this law works, that is, we must know that mediums such as air or water generate a resistance to the movement of bodies, and that this resistance is also associated with the size and weight of the object in free fall. The formula is accepted because we know the domain in which it is accurate, and because our assertions are framed within that domain. Therefore, in order for the law to be accepted we must know the domain in which this law works. In other words, we must examine whether the assumptions that define the domain of the law are realistic or not.

Finally, let us consider the example Friedman mentions about the production of cigarettes and its relationship with supply and demand. Friedman wonders what would happen with the price of cigarettes if a tax were applied on these. General equilibrium theory holds that, under perfect competition, this tax would cause an increase in production costs, which would retract the global supply. This situation would turn worse if the government imposed maximum prices: at such prices the supply would be lower-compared to the equilibrium price-causing a shortage of cigarettes. This was not what happened during the Second World War: the common strength of the increased costs operated with less force than the destructive force of the desire of each company to maintain their portion of the market, the value and prestige of their factory name. According to Friedman, because of this cigarette companies cannot be treated as if they were perfect competitors. His point is that we cannot say whether a given unrealisticness matters before we know what the model is used for. However, this analysis brings about two problems. On the one hand, we are not able to know ex-ante if a theory is good or not until the phenomenon has occurred. Therefore, this criterion does not allow to differentiate between good and bad theories, since we do not know anything about their ability to predict. On the other hand, there is the problem of understanding statements in trend terms. That a theory works under a "wide variety of conditions" does not say anything about its operation in the future. The alternative is to consider that there are different scenarios, and that they lead to different results. Therefore, the important thing is not to consider predictive capacity, but the degree of similarity between the target system and the models that are intended to represent it. During the Second World War the cigarette industry entrepreneurs were thinking in terms of maintaining the status of their factories. This scenario differs from one in which the mechanisms of perfect competition prevails. An investigation that revealed that attitude in businessmen would have allowed economists to make more accurate inferences than those based solely on general equilibrium theory. It is necessary to keep in mind that there are specific characteristics in a target system that determine its results. Once known, they can be used to set a demarcation between which models or theories represent this situation and which do not.

As it can be seen, the realism of the assumptions does matter, because it is the comparison between these and the characteristics of the real world what determines the course of action of a decision maker. Once the idea that we can turn into Russell's turkeys-if we rely solely and exclusively on the knowledge of the past-is accepted, the predictive accu- 
racy choice criterion of models breaks down. Following Taleb (2007), more than pretending to make accurate predictions we may get the idea of future possible scenarios. In this juncture, the choice of models will depend on what the current structure of the target system is. Model inferences are not the benchmark for choosing among them. In any case, these inferences allow us to understand what would happen if the model's structure resembles the structure of the selected part of the world.

\section{Concluding remarks}

In this paper two critiques to Friedman's approach have been presented. On the one hand, there is a difference between a non-direct test and a non-independent test. In some cases, we need to test the model's implications in order to determine whether or not an assumption $S$ is a good approximation to reality. But we may also wonder if an assumption $S$ is more realistic than an assumption $S^{*}$. Perhaps both tests are indirect. However, this does not imply that they are not independent from the test of the model's implications. A clear example of this is the assumption of vacuum in Galileo's law. We may wonder if the statement "the atmospheric pressure is 15 psi" is more realistic than the assumption of vacuum. Clearly it is, and we do not need to examine the predictions of Galileo's law in order to reach such a conclusion. The test is indirect (since we must build a Torricelli experiment-or something similar-for measuring the atmospheric pressure), but it is independent from the test of the implications of Galileo's law.

The other critique is associated with the use of invariant knowledge. It has been shown that Friedman's defense of the unrealism of assumptions only makes sense when the model used for making predictions is grounded on some invariant regularity or stable factor. Yet this is not the case of economic phenomena. At the ontological level, it has been argued that economic phenomena do not respond to the logic of invariance but to a logic of possibility trees or open-ended results. At the epistemological level, it was asserted that predictions based on knowledge from the past involve not only external validity problems but also negative exposures to "black swans". Contrary to this, an approach where the choice between different models depends on the degree of resemblance between the model's structure and the structure of the target system has been proposed. And the way to recognize such resemblance is not by focusing on the predictive accuracy but on the accuracy of the substantive assumptions. Model implications are not-unlike what Friedman claimedthe criterion to evaluate economic models. In any case, these implications will be useful for estimating the possible consequences of an exogenous shock on the target system.

Therefore, the realism of assumptions does turn out to be relevant for evaluating economic models. It is true that several assumptions may turn out to be unrealistic. Nevertheless, we should keep in mind what meaning of "unrealistic" we are using. Claiming that all assumptions are unrealistic because they are abstractions or idealizations does not contribute to the discussion. Labeling any model that makes use of heuristic assumptions as "unrealistic" is not a constructive criticism either. What may shed light is, on the contrary, an evaluation of the realism of the substantive assumptions according to the degree of resemblance with the target system. Once such resemblance is examined, we may have a better view of what models will be useful when making predictions. 


\section{REFERENCES}

Cartwright, Nancy. 1999. The dappled world. Cambridge: Cambridge University Press.

- 2007. Hunting causes and using them - Approaches in philosophy and economics. Cambridge: Cambridge University Press.

Cartwright, Nancy and S. Efstathiou. 2011. Hunting causes and using them: Is there no bridge from here to there? International Studies in the Philosophy of Science 25/3: 223-241.

Friedman, Milton. 1953. Essays in positive economics. Chicago: University of Chicago Press.

Gonzalez, Wenceslao. 2015. Philosophico-methodological analysis of prediction and its role in economics. New York: Springer.

Hands, Douglas. 2001. Reflection without rules. Economic methodology and contemporary science theory. Cambridge: Cambridge University Press.

Hempel, Carl. 1966. Philosophy of natural science. New Jersey: Prentice Hall.

Hindriks, Frank. 2005. Unobservability, tractability, and the battle of assumptions. Journal of Economic Methodology 12/3: 383-406.

Hoover, Kevin. 1984. Methodology: A Comment on Frazer and Boland, II. The American Economic Review 74/4: 789-792.

Ivarola, Leonardo. 2017. Socioeconomic processes as open-ended results. Beyond invariance knowledge for interventionist purposes. Theoria: An International Journal for Theory, History and Foundations of Science 32/2: 211-229.

Keynes, John. 1936. The general theory of employment, interest and money. India: Atlantic.

Kuorikoski, Jaakko and Aki Lehtinen. 2009. Incredible worlds, credible results. Erkenntnis 70/1: 119-31.

Machamer, Peter. 2004. Activities and causation: the metaphysics and epistemology of mechanisms. International Studies in the Philosophy of Science 18/1:27-39.

Machamer, Peter, Lindley Darden and Carl Craver. 2000. Thinking about mechanisms. Philosophy of Science 67/1: 1-25.

Mäki, Uskali. 1992. On the method of idealization in economics. Poznan Studies in the Philosophy of the Sciences and the Humanities 26: 319-354.

-. 2003. 'The methodology of positive economics' (1953) does not give us the methodology of positive economics. Journal of Economic Methodology 10/4: 495-505.

-. 2009a. (ed.) The methodology of positive economics: Reflections on the Milton Friedman legacy. Cambridge: Cambridge University Press.

-. 2009b. Unrealistic assumptions and unnecessary confusions: Rereading and rewriting F53 as a realist statement. In Mäki, Uskali, ed., The methodology of positive economics: Reflections on the Milton Friedman legacy, 90-116. Cambridge: Cambridge University Press.

Musgrave, Alan. 1981. Unreal assumptions in economic theory: The F-twist untwisted. Kyklos 34/3: 377387.

Nagel, Ernest. 1963. Assumptions in economic theory. The American Economic Review 53/2: 211-219.

Taleb, Nassim. 2007. The black swan: The impact of the highly improbable. New York: Random House.

Woodward, James. 2003. Making things happen: A theory of causal explanation. Oxford: Oxford University Press.

Leonardo Ivarola has a BA in Economics and a Ph.D. in Philosophy, both at the University of Buenos Aires. He is currently assistant professor of "epistemology of the economy" at the University of Buenos Aires. He is assistant researcher at the Interdisciplinary Institute of Political Economy of Buenos Aires (University of Buenos Aires/CONICET).

AdDress: Universidad de Buenos Aires, Centro de investigaciones en epistemología de las ciencias económicas (CIECE)/ Consejo Nacional de Investigaciones Científicas y Técnicas (CONICET). Buenos Aires, Argentina. e-mail: I.ivarola@conicet.gov.ar 